\title{
Optimal Dropout Compensation in Networked Control Systems
}

\author{
Qiang Ling and Michael D. Lemmon* \\ Dept. of Electrical Engineering \\ University of Notre Dame
}

March 7, 2003

\begin{abstract}
This paper examines optimal compensation for dropped feedback measurements in a networked control system. A common policy for handling such lost data is to simply use the past data. This case was treated in [8]. This paper extends that prior work to cover a more general class of dropout compensation. The paper's principal result shows that determining the optimal dropout compensator can be posed as a constrained generalized regulator problem. An example compares the performance of a networked control system using the optimal dropout compensator against more commonly used heuristic dropout policies. The comparison shows that the optimal compensator works better than these heuristic policies.
\end{abstract}

\section{Introduction}

Networked control systems (NCS) are control systems whose feedback paths are implemented by a communication network. The communication network can severely degrade overall control system performance through quantization errors and dropped measurements. Quantization errors occur when a sensor measurement is encoded into a fixed number of bits. Measurements are dropped when the channel causes transmission errors that force the receiver to drop the entire measurement packet. In both cases, we find the total information that can be transmitted between the control system's sensor and actuator is limited. A great deal of recent work has focused on the effect of quantization errors on overall closed loop system performance under the assumption that the channel is inherently reliable [2] [4] [1] [3]. This paper studies the effect that unreliable channels have on overall system performance assuming that there are no quantization errors.

There has been a small amount of work examining the impact of dropouts in networked control systems. In [10], the performance of the system as measured by the $\mathcal{H}_{\infty}$ gain was expressed as a function of packet loss. Similar results were obtained in [8] and [9] in which the power semi-norms of output signals were computed under certain statistical dropout models. In [6], networked control systems with dropouts are modelled as asynchronous switched systems. The approach replaces the true switched system with an "averaged" system and then studies the stability of this system. In [5], the dropouts are governed by a Markov chain and are treated as "vacant" sampling. The work proposes two different compensation schemes, keeping the old

\footnotetext{
*We gratefully acknowledge the partial financial support of the National Science Foundation (NSF-CCR02-8537) and the Army Research Office (DAAD19-01-0743)
} 
control or generating new control with the estimation of the lost data. Then the stability of an LQ controller under these schemes is informally analyzed. Recent work has examined the performance of networked control systems under dropouts. An analysis of such systems is found in [7] in which dropped measurements are replaced by zeros. A more complete analysis of dropouts for single-input single-output control system will be found in [8] for dropout processes that are i.i.d. (identically independently distributed) in nature. In this paper, dropout compensation involved using the last received sample. A formal extension of that work to dropout processes governed by Markov chains will be found in [9]. In these papers, although some heuristic dropout compensators are used, there is little discussion on choosing dropout compensator.

This paper formally derives a compensator for data dropouts that optimizes overall control system performance. If we let $y$ denote the control system's output signal, then control system performance is measured by the power semi-norm $\|y\|_{\mathcal{P}}=\sqrt{\operatorname{Trace}\left(R_{y y}(0)\right)}$ where $R_{y y}[0]$ is the covariance of $y$. We extend the prior work in [8] to derive the power spectral density (PSD) of $y$ when a generalized dropout compensation policy is used. Our characterization of the PSD shows that we can pose an "optimal" dropout compensation problem, in which we find the policy that optimizes overall control system performance. What is interesting here is that the optimization problem takes the form of a constrained generalized regulator problem.

The remainder of this paper is organized as follows. Section 2 presents mathematical preliminaries. Section 3 presents a model for the networked control system. Section 4 states a closed form expression for the PSD of the closed loop system's output signal as a function of the dropout rate and dropout compensation policy. This result is an extension of our earlier work in [8]. An equivalent linear time-invariant model for our NCS is presented in section 5. Section 6 uses this equivalent model to state an optimization problem whose solution is the "optimal" dropout compensator. Final comments are presented in section 7. Proofs of the two major results in the paper will be found in sections 8 and 9 .

\section{Mathematical Preliminaries}

Let $x$ be a random vector and let $\mathbf{E}[x]$ denote the expectation of $x$. A real-valued discrete-time stochastic process $x=\{x[n]\}$ is convergent in the mean square sense if there exists a random vector $\bar{x}$ such that

$$
\lim _{n \rightarrow \infty} \mathbf{E}\left[(x[n]-\bar{x})^{T}(x[n]-\bar{x})\right]=0 .
$$

It can be shown [11] that a random process $x=\{x[n]\}$ is convergent in the mean square sense if and only if

$$
\lim _{n \rightarrow \infty} \sup _{m \geq n} \mathbf{E}\left[(x[m]-x[n])^{T}(x[m]-x[n])\right]=0
$$

A jump linear system is a linear dynamical system whose system matrices are random processes. It has the form

$$
\left\{\begin{array}{ccc}
x[n+1] & =A[n] x[n]+B[n] w[n] \\
y[n] & = & C[n] x[n]+D[n] w[n]
\end{array},\right.
$$

where $x=\{x[n]\}$ is the system's state process, $w=\{w[n]\}$ is a random process representing an exogenous input, $y=\{y[n]\}$ is the system's output process, and $\{A[n]\},\{B[n]\},\{C[n]\}$, and $\{D[n]\}$ are matrix valued random processes. If the input process $w[n]=0$, then we say that the system is a free jump linear system. A free jump linear system is said to be asymptotically stable in the mean square sense [15] whenever

$$
\lim _{n \rightarrow \infty} \mathbf{E}\left[x[n]^{T} x[n] \mid x_{0}\right]=0
$$


for any initial states $x[0]=x_{0}$.

This paper confines its attention to the jump linear systems that are strictly proper single-input single-output, i.e. $y[n], u[n]$ are scalar and $D[n]=0$.

A random process $x=\{x[n]\}$ is said to be wide sense stationary (WSS) if and only if $\mathbf{E}[x[n]]=$ constant $=$ $\mu_{x}$ and $\mathbf{E}\left[\left(x[k]-\mu_{x}\right)\left(x[l]-\mu_{x}\right)^{T}\right]=\mathbf{E}\left[\left(x[k+n]-\mu_{x}\right)\left(x[l+n]-\mu_{x}\right)^{T}\right]$ for arbitrary $n$. The mean of the WSS process $x=\{x[n]\}$ is denoted as $\mu_{x}$ and the correlation matrix of this process is denoted as $R_{x x}(m)=\mathbf{E}\left[x[n+m] x[n]^{T}\right]$.

If $x=\{x[n]\}$ is WSS, then the power spectral density (PSD) of $x$ and its covariance function are related through the equations,

$$
S_{x x}(z)=\sum_{k=-\infty}^{\infty} R_{x x}(m) z^{-k}, \quad R_{x x}[0]=\frac{1}{2 \pi} \int_{-\pi}^{\pi} S_{y y}\left(e^{j \omega}\right) d \omega .
$$

Most of the WSS processes in this paper are zero mean, so their covariances (cross-covariances) and correlations (cross-correlations) are equal. We interchangeably use these terms throughout the paper.

Some of the technical proofs in section 8 use a single-sided Z transform. Given a WSS process $x$, the positive and negative single-sided power spectral densities are defined by the equations

$$
S_{x x}^{+}(z)=\sum_{m=1}^{\infty} R_{x x}[m] z^{-k}, \quad S_{x x}^{-}(z)=\sum_{m=-\infty}^{-1} R_{x x}[m] z^{-k} .
$$

So the power spectral density can be expressed as

$$
S_{x x}(z)=S_{x x}^{+}(z)+S_{x x}^{-}(z)+R_{x x}[0] .
$$

Since $R_{x x}(m)=R_{x x}^{T}(-m)$, it is straightforward to see that $S_{x x}^{+}(z)=\left[S_{x x}^{-}\left(z^{-1}\right)\right]^{T}$.

Some of the technical proofs in section 9 make use of the Kronecker product, $\otimes$. The Kronecker product of two matrices $A=\left(a_{i j}\right)_{M \times N}, B=\left(b_{p q}\right)_{P \times Q}$ is defined as

$$
A \otimes B=\left[\begin{array}{cccc}
a_{11} B & a_{12} B & \cdots & a_{1 N} B \\
a_{21} B & a_{22} B & \cdots & a_{2 N} B \\
\vdots & \vdots & \ddots & \vdots \\
a_{M 1} B & a_{M 2} B & \cdots & a_{M N} B
\end{array}\right]
$$

For simplicity, $A \otimes A$ is denoted as $A^{[2]}$ and $A \otimes A^{[n]}$ is denoted as $A^{[n+1]}(n \geq 2)$. The following property of Kronecker product will be used in the technical proofs,

$$
\left(A_{1} A_{2} \cdots A_{n}\right) \otimes\left(B_{1} B_{2} \cdots B_{n}\right)=\left(A_{1} \otimes B_{1}\right)\left(A_{2} \otimes B_{2}\right) \cdots\left(A_{n} \otimes B_{n}\right),
$$

where $A_{i}, B_{i}(i=1,2, \cdots, n)$ are all matrices with appropriate dimensions. In the computations on Kronecker product, two linear operators, vec and devec, are used. They are defined as follows.

Definition 2.1 vec $i$ s applied to a matrix $A=\left(a_{i j}\right)_{M \times N} \cdot \operatorname{vec}(A)=\left[\begin{array}{lllll}a_{11} & a_{21} \cdots a_{M 1} & a_{12} & \cdots & a_{M 2} \\ \cdots & a_{1 N} & \cdots & a_{M N}\end{array}\right]^{T}$.

Definition 2.2 devec is the inverse of vec for a square matrix. When $A$ is square, devec $(\operatorname{vec}(A))=A$. 


\section{Network Control System Model}

We consider a networked control system in which $H(z)$ is a strictly proper loop function. The control system uses unity gain feedback in which the loop function's output $y$ is fed back through a binary erasure channel. A block diagram of this system is shown in figure 1. The channel drops a transmitted measurement with a probability $\epsilon$. The channel, therefore, may be modelled as a system that takes the signal $y$ and a random dropout signal $d$ as inputs. The dropout process $d$ is a binary random process that takes the value 1 if a dropout occurs and is zero otherwise. The channel produces an output $y^{\prime}[n]$ that is equal to $y[n]$ when $d[n]=0$ and is zero otherwise. A linear filter, $F(z)$, is used to correct for dropped measurements. $F(z)$ is also strictly proper. The input to the dropout compensator is the system's output measurement $y[n]$ (i.e. when there are no dropouts) or it is a prediction of the system output, $\hat{y}[n]$. The prediction is generated by the linear filter $F(z)$ as shown in the figure. The signal, $\bar{y}[n]$, used by the closed loop system equals the true plant output, $y[n]$ when there is no dropout and switches to the predicted plant output, $\hat{y}[n]$, when a dropout occurs. Figure 1 models the effect of the dropout by multiplying $\hat{y}[n]$ and $y[n]$ by $d[n]$ and the logical inversion of $d[n]$, respectively. Note that our figure denotes the inversion of $d[n]$ with an inversion bubble on the multiplier.

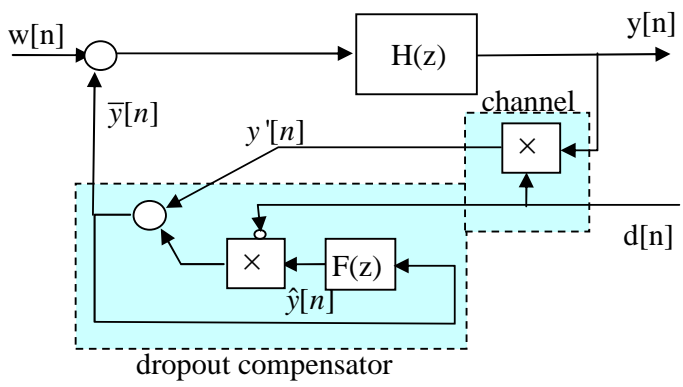

Figure 1: Networked Control System with Data Dropouts and Dropout Compensation

The preceding system can be treated as a jump linear system whose state is denoted as $x=\left[\begin{array}{ll}x_{h}^{T} & x_{f}^{T}\end{array}\right]^{T}$. The subvector $x_{h}$ denotes the state of the loop function $H(z)$ and $x_{f}$ denotes the state of the dropout compensator $F(z)$. Assume that $H$ and $F$ have minimal state space realizations, $H \stackrel{s}{=}\left[\begin{array}{c|c}A_{h} & B_{h} \\ \hline C_{h} & 0\end{array}\right]$ and $F \stackrel{s}{=}\left[\begin{array}{c|c}A_{f} & B_{f} \\ \hline C_{f} & 0\end{array}\right]$. The state equations for the entire NCS are

$$
\left\{\begin{aligned}
x[k+1] & =A[k] x[k]+B w[k] \\
y[k] & =C x[k]
\end{aligned}\right.
$$

where

$$
A[k]=\left\{\begin{array}{cc}
A_{0}=\left[\begin{array}{cc}
A_{h}+B_{h} C_{h} & 0 \\
B_{f} C_{h} & A_{f} \\
A_{h} & B_{h} C_{f} \\
0 & A_{f}+B_{f} C_{f}
\end{array}\right], \quad \text { with probability } 1-\epsilon \\
A_{1}=\text { with probability } \epsilon
\end{array}\right.
$$

and $B=\left[\begin{array}{c}B_{h} \\ 0\end{array}\right]$ and $C=\left[\begin{array}{ll}C_{h} & 0\end{array}\right]$. 
Let $\bar{A}_{[2]}=(1-\epsilon) A_{0}^{[2]}+\epsilon A_{1}^{[2]}$. The matrix $\bar{A}_{[2]}$ is said to be stable if all of its eigenvalues lie within the unit circle. It can be proven using techniques in [9] that the NCS is asymptotically stable in mean square sense if and only if $\bar{A}_{[2]}$ is stable. Since this paper derives the PSD for various signals in the NCS, we must be able to assure that the NCS is wide sense stationary. It was shown in [8] that the NCS is wide sense stationary if $\bar{A}_{[2]}$ is stable and the input signal $w$ is WSS. Throughout this paper, we assume that $\bar{A}_{[2]}$ is stable, so we will not explicitly mention the wide sense stationarity of the signals generated by the NCS.

\section{Power Spectral Density}

The following theorem presents a closed form expression for the PSDs of the output signals $y[n]$ and $\tilde{y}[n]=$ $y[n]-\bar{y}[n]$ generated by the NCS 4 . The result is a generalization of results presented in [8]. The proof will be found in section 8 .

Theorem 4.1 Consider the NCS 4. $w$ is white with zero mean and unit covariance, whose power spectral density is $S_{w w}(z)=1$. The dropout process $d$ is i.i.d. with the probability distribution of $\operatorname{Pr}(d[n]=1)=\epsilon$ and $\operatorname{Pr}(d[n]=0)=1-\epsilon$. $d$ is independent of $w$. Let $\tilde{y}[n]=y[n]-\bar{y}[n]$. If $\bar{A}_{[2]}=(1-\epsilon) A_{0}^{[2]}+\epsilon A_{1}^{[2]}$ is stable,

$$
\begin{aligned}
S_{y y}(z) & =\left|\frac{H(z)}{1-D(z) H(z)}\right|^{2} S_{w w}(z)+\left|\frac{D(z) H(z)}{1-D(z) H(z)}\right|^{2} \frac{1}{1-\epsilon} \Delta \\
S_{\tilde{y} \tilde{y}}(z) & =\left|\frac{H(z)(D(z)-1)}{1-D(z) H(z)}\right|^{2} S_{w w}(z)+\left|\frac{D(z)(1-H(z))}{1-D(z) H(z)}\right|^{2} \frac{1}{1-\epsilon} \Delta, \\
\|y\|_{\mathcal{P}} & =\frac{1}{2 \pi} \int_{-\pi}^{\pi} S_{y y}\left(e^{j \omega}\right) d \omega
\end{aligned}
$$

where $|\cdot|$ means magnitude, $D(z)=\frac{1-\epsilon}{1-\epsilon F(z)}, \Delta=R_{\tilde{y} \tilde{y}}[0]$. When $\bar{A}_{[2]}$ is stable and $\epsilon>0{ }^{1}$,

$$
\frac{1}{2 \pi} \int_{-\pi}^{\pi}\left|\frac{D\left(e^{j \omega}\right)\left(1-H\left(e^{j \omega}\right)\right)}{1-D\left(e^{j \omega}\right) H\left(e^{j \omega}\right)}\right|^{2}<1-\epsilon
$$

and $\Delta=R_{\tilde{y} \tilde{y}}[0]$ is the unique nonnegative solution of the following equation

$$
\Delta=\frac{1}{2 \pi} \int_{-\pi}^{\pi}\left|\frac{H\left(e^{j \omega}\right)\left(D\left(e^{j \omega}\right)-1\right)}{1-D\left(e^{j \omega}\right) H\left(e^{j \omega}\right)}\right|^{2} S_{w w}\left(e^{j \omega}\right) d \omega+\frac{1}{2 \pi} \int_{-\pi}^{\pi}\left|\frac{D\left(e^{j \omega}\right)\left(1-H\left(e^{j \omega}\right)\right)}{1-D\left(e^{j \omega}\right) H\left(e^{j \omega}\right)}\right|^{2} d \omega \frac{1}{1-\epsilon} \Delta
$$

\section{Equivalent NCS Model}

The power spectral densities stated in theorem 4.1 can also be generated by a linear feedback system. Figure 2 is a block diagram of this equivalent system. It is a classical control loop with two inputs, the unit variance white disturbance noise $\{w[n]\}$ and an equivalent white "dropout" noise process $\{n[n]\}$ with variance $\Delta /(1-\epsilon)$ where

$$
\Delta=R_{\tilde{y} \tilde{y}}[0]
$$

\footnotetext{
${ }^{1}$ When $\epsilon=0, \tilde{y}[n]=0$. So $R_{\tilde{y} \tilde{y}}[0]=0$.
} 
From the state space realizations of $H(z)$ and $D(z)$, we can see that our equivalent system has the state space realization.

$$
\left\{\begin{aligned}
x_{e}[k+1] & =A_{e} x_{e}[k]+B_{w} w[k]+B_{n} n[k] \\
y[k] & =C_{y} x_{e}[k] \\
\tilde{y}[k] & =C_{\tilde{y}} x_{e}[k]+(1-\epsilon) n[k]
\end{aligned}\right.
$$

where $x_{e}=\left[\begin{array}{ll}x_{h}^{T} & x_{f}^{T}\end{array}\right]^{T}, A_{e}=(1-\epsilon) A_{0}+\epsilon A_{1}, B_{w}=\left[\begin{array}{c}B_{h} \\ 0\end{array}\right], B_{n}=(1-\epsilon)\left[\begin{array}{c}B_{h} \\ B_{f}\end{array}\right], C_{y}=\left[\begin{array}{ll}C_{h} & 0\end{array}\right], C_{\tilde{y}}=$ $\epsilon\left[\begin{array}{ll}-C_{h} & C_{f}\end{array}\right]$.

Note that the covariance of the noise process, $n$, is dependent on the output $\tilde{y}$. So the above control system is well-posed only if we can assure that eq. 10 has a unique nonnegative solution with respect to $\Delta$. The following theorem establishes the well-posed condition. Furthermore, it establishes the equivalence between the stability of system 4 and 11 and the equivalence between the PSDs generated by the two systems. The proof for this theorem is in section 9 .

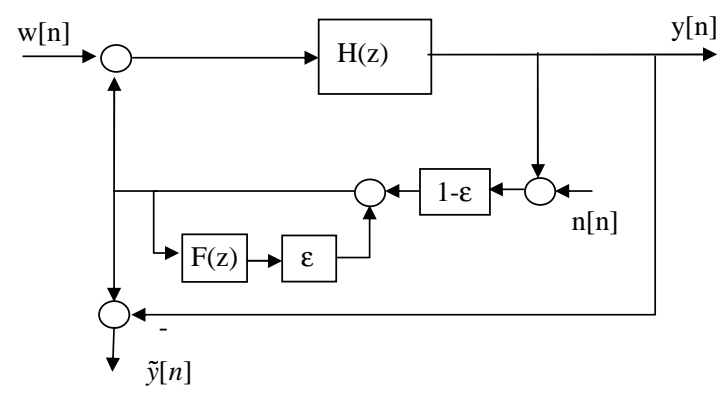

Figure 2: Equivalent system

Theorem 5.1 The system 11 is asymptotically stable and its transfer function $G_{\tilde{y} n}(z)$ satisfies

$$
\left\|G_{\tilde{y} n}\right\|_{2}^{2}<1-\epsilon
$$

if and only if the system 4 is asymptotically stable in mean square sense.

When system 11 is asymptotically stable and the constraint 12 is satisfied, eq. 10 has a unique nonnegative solution.

Furthermore, when the two systems are stable, they will generate the same power spectral densities, $S_{y y}(z)$ and $S_{\tilde{y} \tilde{y}}(z)$.

\section{Optimal Dropout Compensator Design}

Our goal is to design $F(z)$ to minimize $R_{y y}[0]=\frac{1}{2 \pi} \int_{-\pi}^{\pi} S_{y y}\left(e^{j \omega}\right) d \omega$ when the system 4 is asymptotically stable in mean square sense. By theorem 5.1, this goal is equivalent to minimizing $R_{y y}[0]$ for the equivalent system 11 under the constraints that 
- the system 11 is asymptotically stable

- and $\left\|G_{\tilde{y} n}(z)\right\|_{2}^{2}<1-\epsilon$;

This means that a compensator for the original system (eq. 4) can be obtained by solving a controller synthesis problem for a linear system with a constraint. This section provides a statement of that synthesis problem and then solves it for an explicit example using a brute-force optimization method.

To solve the problem, we must compute $R_{y y}[0]$ and $\left\|G_{\tilde{y} n}(z)\right\|_{2}^{2}$. These objects can be computed from the system's controllability Gramian using standard techniques in linear system theories [14]. In particular, we can represent these objects by the following equations,

$$
\left\{\begin{aligned}
R_{y y}[0] & =\left\|G_{y w}\right\|_{2}^{2}+\left\|G_{y n}\right\|_{2}^{2} \frac{1}{1-\epsilon} \Delta \\
\Delta & =R_{\tilde{y} \tilde{y}}[0] \\
R_{\tilde{y} \tilde{y}}[0] & =\left\|G_{\tilde{y} w}\right\|_{2}^{2}+\left\|G_{\tilde{y} n}\right\|_{2}^{2} \frac{1}{1-\epsilon} \Delta \\
\left\|G_{y w}\right\|_{2}^{2} & =C_{y} W_{w} C_{y}^{T} \\
\left\|G_{y n}\right\|_{2}^{2} & =C_{y} W_{n} C_{y}^{T} \\
\left\|G_{\tilde{y} w}\right\|_{2}^{2} & =C_{\tilde{y}} W_{w} C_{\tilde{y}}^{T} \\
\left\|G_{\tilde{y} n}\right\|_{2}^{2} & =C_{\tilde{y}} W_{n} C_{\tilde{y}}^{T}+(1-\epsilon)^{2}
\end{aligned}\right.
$$

where $W_{w}$ and $W_{n}$ are the controllability Gramian matrices that must satisfy the Riccati equations

$$
\left\{\begin{aligned}
A_{e} W_{w} A_{e}^{T}+B_{w} B_{w}^{T} & =W_{w} \\
A_{e} W_{n} A_{e}^{T}+B_{n} B_{n}^{T} & =W_{n}
\end{aligned}\right.
$$

Our performance index $R_{y y}[0]$ and constraint $\left\|G_{\tilde{y} n}\right\|_{2}^{2}$ are all functions of the parameters of system 11, i.e. $A_{e}, B_{w}, B_{n}, C_{y}, C_{\tilde{y}}$. System 11 is the combination of $H(z)$ and $F(z)$, and $H(z)$ is a known plant, so $A_{e}, B_{w}, B_{n}, C_{y}, C_{\tilde{y}}$ will depend on only the parameters of $F(z)$, i.e. $A_{f}, B_{f}, C_{f}$. Then $R_{y y}[0]$ and $\left\|G_{\tilde{y} n}\right\|_{2}^{2}$ are functions of $A_{f}, B_{f}, C_{f}$. By searching $A_{f}, B_{f}, C_{f}$, we may find the optimal (minimal) $R_{y y}[0]$ with constraint 12 satisfied. In order to do optimization more efficiently, we put the following structural constraint

$$
F(z)=\frac{\sum_{j=1}^{N} b_{j} z^{-j}}{1+\sum_{i=1}^{N} a_{i} z^{-i}}
$$

and we take the controller canonical form realization of $F(z)$, i.e.

$$
A_{f}=\left[\begin{array}{ccccc}
0 & 0 & \cdots & 0 & -a_{N} \\
1 & 0 & \cdots & 0 & -a_{N-1} \\
\vdots & \vdots & \ddots & \vdots & \vdots \\
0 & 0 & \cdots & 1 & -a_{1}
\end{array}\right], B_{f}=\left[\begin{array}{c}
b_{N} \\
b_{N-1} \\
\vdots \\
b_{1}
\end{array}\right], C_{f}=\left[\begin{array}{llll}
0 & \cdots & 0 & 1
\end{array}\right]
$$

where $b_{j} \in \mathcal{R}, a_{i} \in \mathcal{R}(i, j=1,2, \cdots, N)$. We will really search $b_{j}, a_{i}(i, j=1,2, \cdots, N) . A_{e}, B_{n}$ are all linear functions with respect to $b_{j}, a_{i} ; B_{w}, C_{y}, C_{\tilde{y}}$ are constants with respect to $b_{j}, a_{i}$. Then we can formulate our optimization problem as following. 


\section{Optimization 6.1}

$$
\begin{array}{cl}
\text { minimize: } & C_{y} W_{w} C_{y}^{T}+C_{y} W_{n} C_{y}^{T} \frac{1}{1-\epsilon} \Delta, \\
\text { with respect to: } & b_{j}, a_{i}(i, j=1,2, \cdots, N) \\
\text { subject to: } & C_{\tilde{y}} W_{n} C_{\tilde{y}}^{T}<\epsilon(1-\epsilon) \\
& \Delta=\frac{(1-\epsilon) C_{\tilde{y}} W_{w} C_{\tilde{y}}^{T}}{\epsilon(1-\epsilon)-C_{\tilde{y}} W_{n} C_{\tilde{y}}^{T}} \\
& A_{e} W_{w} A_{e}^{T}+B_{w} B_{w}^{T}=W_{w} \\
& A_{e} W_{n} A_{e}^{T}+B_{n} B_{n}^{T}=W_{n}
\end{array}
$$

As an example, we took the plant to be $H(z)=\frac{z^{-1}+2 z^{-2}}{1+z^{-1}+2 z^{-2}}$. Optimization 6.1 is solved with the MATLAB nonlinear optimization toolbox fmincon() for $N=2$ and $\epsilon \in[0,0.24]$ (Remark: note that the problem is infeasible when $\epsilon>24.9 \%$ ). The performance, $R_{y y}[0]$, achieved using the "optimal" dropout compensation filter was compared against the performance of other popular compensators. In particular, we used the compensator $F(z)=z^{-1}$ (used in [8]) and the compensator $F(z)=0$ (used in [7]). Figure 3 plots the performance $R_{y y}[0]$ as a function of the dropout rate $\epsilon$ for these three dropout compensators. The figure shows that our optimal compensator indeed outperforms both of the popular heuristic dropout schemes.

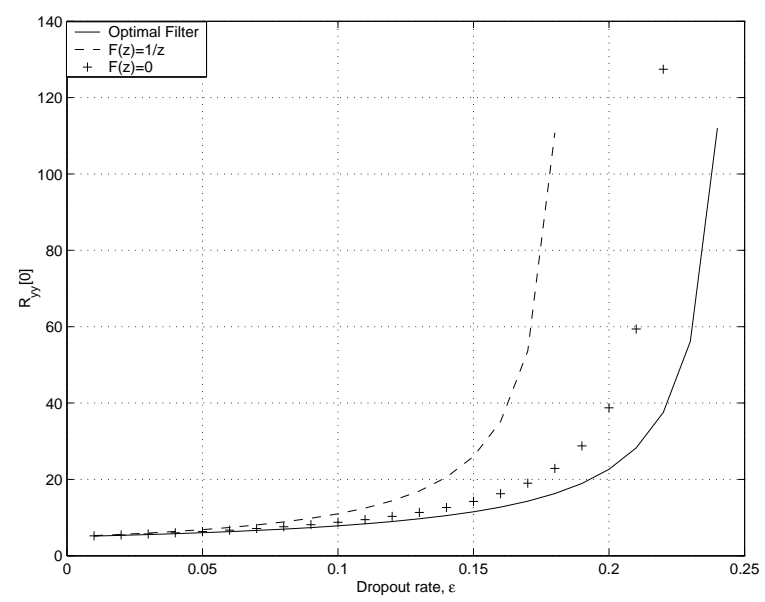

Figure 3: Performance comparation under different dropout compensators

\section{Conclusions}

This paper analyzes a networked control system with a general linear dropout compensator under i.i.d. dropouts. A closed form expression of the output's PSD is achieved. Based on the PSD, the original networked control system is converted into an equivalent traditional linear system with a constraint. Therefore the dropout compensator design problem converts the problem of finding optimal dropout policies into a constrained generalized regulator problem. Although this paper studies only the single-input single-output plants, all the results can be easily extended to the multiple-input multiple-output cases.

The optimization problem in this paper takes a nonlinear form. If we can transform it into a linear form, the powerful linear optimization tools can be utilized and the computation efficiency can be greatly improved. 
Furthermore, if the optimal dropout compensator can be designed directly rather than through optimization, the best performance can be achieved and the tightest bound for the compensator optimization can be put.

In this paper, an equivalent linear system with a constraint is proposed, which is a powerful tool for studying NCSs. Here we use it to design dropout compensator. Some other objectives, such as optimal controller design for an NCS, can be achieved with the equivalent system. So it may deserve to further exploit the equivalent system's potentials.

\section{Proof to Theorem 4.1}

When we compute PSDs, the impulse response descriptions of linear systems will be useful. Let us assume the impulse responses of $H(z)$ and $F(z)$ are respectively $h$ and $f$, i.e.

$$
\begin{aligned}
& y=h *(\bar{y}+w) \\
& \hat{y}=f * \bar{y}
\end{aligned}
$$

where $*$ denotes convolution.

The proofs in this section assume that the dropout process $d$ is i.i.d., that the processes $d$ and $w$ are statistically independent, and that $H(z)$ and $F(z)$ are strictly proper. The strictly proper assumption assures that $y[n], \hat{y}[n], \bar{y}[n]$ and $\tilde{y}[n]$ depend on only the last dropouts and the last input noises, i.e. $\{d[n-$ $1], d[n-2], \cdots\}$ and $\{w[n-1], w[n-2], \cdots\}$. The following proofs are stated without explicitly stating these assumptions.

The proof to theorem 4.1 is quite invovled. So we break it into several steps. Every step computes a couple of PSDs, which together will lead to the final results.

\subsection{Computing $S_{y w}(z), S_{\bar{y} w}(z)$ and $S_{\hat{y} w}(z)$}

By $y=h *(\bar{y}+w)$, we get

$$
S_{y w}(z)=H(z)\left(S_{\bar{y} w}(z)+S_{w w}(z)\right)
$$

For any $m$, we get

$$
\begin{aligned}
R_{\bar{y} w}[m] & =\mathbf{E}[\bar{y}[n+m] w[n]] \\
& =\mathbf{E}[\bar{y}[n+m] w[n] \mid d[n+m]=0] \operatorname{Pr}(d[n+m]=0)+\mathbf{E}[\bar{y}[n+m] w[n] \mid d[n+m]=1] \operatorname{Pr}(d[n+m]=1) \\
& =\mathbf{E}[y[n+m] w[n]] \operatorname{Pr}(d[n+m]=0)+\mathbf{E}[\hat{y}[n+m] w[n]] \operatorname{Pr}(d[n+m]=1) \\
& =(1-\epsilon) R_{y w}[m]+\epsilon R_{\hat{y} w}[m]
\end{aligned}
$$

So we get

$$
S_{\bar{y} w}(z)=(1-\epsilon) S_{y w}(z)+\epsilon S_{\hat{y} w}(z)
$$

By $\hat{y}=f * \bar{y}$, we get

$$
S_{\hat{y} w}=F(z) S_{\bar{y} w}
$$


Combine equations 20, 21 and 22, we get

$$
\begin{aligned}
S_{\bar{y} w}(z) & =\frac{D(z) H(z)}{1-D(z) H(z)} S_{w w}(z) \\
S_{y w}(z) & =\frac{H(z)}{1-D(z) H(z)} S_{w w}(z) \\
S_{\hat{y} w}(z) & =\frac{D(z) H(z) F(z)}{1-D(z) H(z)} S_{w w}(z)
\end{aligned}
$$

where $D(z)=\frac{1-\epsilon}{1-\epsilon F(z)}$.

\subsection{Computations on $S_{\bar{y} \hat{y}}(z)$}

\subsubsection{Computing $S_{\bar{y} \hat{y}}^{+}(z)$}

When $m>0$, we get

$$
\begin{aligned}
R_{\bar{y} \hat{y}}[m] & =\mathbf{E}[\bar{y}[n+m] \hat{y}[n]] \\
& =\mathbf{E}[\bar{y}[n+m] \hat{y}[n] \mid d[n+m]=0] \operatorname{Pr}(d[n+m]=0)+\mathbf{E}[\bar{y}[n+m] \hat{y}[n] \mid d[n+m]=1] \operatorname{Pr}(d[n+m]=1) \\
& =\mathbf{E}[y[n+m] \hat{y}[n]] \operatorname{Pr}(d[n+m]=0)+\mathbf{E}[\hat{y}[n+m] \hat{y}[n]] \operatorname{Pr}(d[n+m]=1) \\
& =(1-\epsilon) R_{y \hat{y}}[m]+\epsilon R_{\hat{y} \hat{y}}[m]
\end{aligned}
$$

By the definition of the single-sided PSD, we get

$$
S_{\bar{y} \hat{y}}^{+}(z)=(1-\epsilon) S_{y \hat{y}}^{+}(z)+\epsilon S_{\hat{y} \hat{y}}^{+}(z)
$$

\subsubsection{Computing $S_{\bar{y} \hat{y}}^{-}(z)$}

When $m<0$, we get

$$
\begin{aligned}
R_{\overline{y y}}[m] & =\mathbf{E}[\bar{y}[n+m] \bar{y}[n]] \\
& =\mathbf{E}[\bar{y}[n+m] \bar{y}[n] \mid d[n]=0] \operatorname{Pr}(d[n]=0)+\mathbf{E}[\bar{y}[n+m] \bar{y}[n] \mid d[n]=1] \operatorname{Pr}(d[n]=1) \\
& =\mathbf{E}[\bar{y}[n+m] y[n]] \operatorname{Pr}(d[n]=0)+\mathbf{E}[\bar{y}[n+m] \hat{y}[n]] \operatorname{Pr}(d[n]=1) \\
& =(1-\epsilon) R_{\bar{y} y}[m]+\epsilon R_{\bar{y} \hat{y}}[m]
\end{aligned}
$$

Then

$$
R_{\bar{y} \hat{y}}[m]=\frac{1}{\epsilon} R_{\overline{y y}}[m]-\frac{1-\epsilon}{\epsilon} R_{\bar{y} y}[m]
$$

By the definition of the single-sided PSD, we get

$$
S_{\bar{y} \hat{y}}^{-}(z)=\frac{1}{\epsilon} S_{\overline{y y}}^{-}(z)-\frac{1-\epsilon}{\epsilon} S_{\bar{y} y}^{-}(z)
$$




\subsubsection{Computing $S_{\bar{y} \hat{y}}(z)$}

By $\hat{y}=f * \bar{y}$, we get

$$
S_{\bar{y} \hat{y}}(z)=F\left(z^{-1}\right) S_{\overline{y y}}(z)
$$

By the definitions of the single-sided PSDs, we get

$$
S_{\bar{y} \hat{y}}(z)=S_{\bar{y} \hat{y}}^{+}(z)+S_{\bar{y} \hat{y}}^{-}(z)+R_{\bar{y} \hat{y}}[0]
$$

Substitute eq. 26,27 and 28 into eq. 29 , we get

$$
(1-\epsilon) S_{y \hat{y}}^{+}(z)+\epsilon S_{\hat{y} \hat{y}}^{+}(z)+\frac{1}{\epsilon} S_{\overline{y y}}^{-}(z)-\frac{1-\epsilon}{\epsilon} S_{\bar{y} y}^{-}(z)+R_{\bar{y} \hat{y}}[0]=F\left(z^{-1}\right) S_{\overline{y y}}(z)
$$

From the above equation, we get

$$
S_{\bar{y} y}^{-}(z)=\frac{\epsilon}{1-\epsilon}\left[(1-\epsilon) S_{y \hat{y}}^{+}(z)+\epsilon S_{\hat{y} \hat{y}}^{+}(z)+\frac{1}{\epsilon} S_{\overline{y y}}^{-}(z)+R_{\bar{y} \hat{y}}[0]-F\left(z^{-1}\right) S_{\overline{y y}}(z)\right]
$$

\subsection{Computations on $S_{\bar{y} y}(z)$}

In the above subsection, we have already got $S_{\bar{y} y}^{-}(z)$. So only $S_{\bar{y} y}^{+}(z)$ needs to compute.

\subsubsection{Computing $S_{\bar{y} y}^{+}(z)$}

When $m>0$,

$$
\begin{aligned}
R_{\bar{y} y}[m] & =\mathbf{E}[\bar{y}[n+m] y[n]] \\
& =\mathbf{E}[\bar{y}[n+m] y[n] \mid d[n+m]=0] \operatorname{Pr}(d[n+m]=0)+\mathbf{E}[\bar{y}[n+m] y[n] \mid d[n+m]=1] \operatorname{Pr}(d[n+m]=1) \\
& =\mathbf{E}[y[n+m] y[n]] \operatorname{Pr}(d[n+m]=0)+\mathbf{E}[\hat{y}[n+m] y[n]] \operatorname{Pr}(d[n+m]=1) \\
& =(1-\epsilon) R_{y y}[m]+\epsilon R_{\hat{y} y}[m]
\end{aligned}
$$

By the definition of the single-sided PSD, we get

$$
S_{\bar{y} y}^{+}(z)=(1-\epsilon) S_{y y}^{+}(z)+\epsilon S_{\hat{y} y}^{+}(z)
$$

\subsubsection{Computing $S_{\bar{y} y}(z)$}

By $y=h *(\bar{y}+w)$, we get

$$
S_{\bar{y} y}(z)=H\left(z^{-1}\right)\left(S_{\overline{y y}}(z)+S_{\bar{y} w}(z)\right)
$$

Again we know that

$$
S_{\bar{y} y}(z)=S_{\bar{y} y}^{+}(z)+S_{\bar{y} y}^{-}(z)+R_{\bar{y} y}[0]
$$


Substitute eq. 32,31 and 30 into eq. 33, we get

$$
\begin{aligned}
& (1-\epsilon) S_{y y}^{+}(z)+\epsilon S_{\hat{y} y}^{+}(z)+\epsilon S_{y \hat{y}}^{+}(z)+\frac{\epsilon^{2}}{1-\epsilon} S_{\hat{y} \hat{y}}^{+}(z)+\frac{1}{1-\epsilon} S_{\overline{y y}}^{-}(z) \\
& -\frac{\epsilon}{1-\epsilon} F\left(z^{-1}\right) S_{\overline{y y}}(z)+\frac{\epsilon}{1-\epsilon} R_{\bar{y} \hat{y}}[0]+R_{\bar{y} y}[0] \\
= & H\left(z^{-1}\right)\left(S_{\overline{y y}}(z)+S_{\bar{y} w}(z)\right)
\end{aligned}
$$

In eq. 34 , we replace $z$ with $z^{-1}$, and get

$$
\begin{aligned}
& (1-\epsilon) S_{y y}^{+}\left(z^{-1}\right)+\epsilon S_{\hat{y} y}^{+}\left(z^{-1}\right)+\epsilon S_{y \hat{y}}^{+}\left(z^{-1}\right)+\frac{\epsilon^{2}}{1-\epsilon} S_{\hat{y} \hat{y}}^{+}\left(z^{-1}\right)+\frac{1}{1-\epsilon} S_{\overline{y y}}^{-}\left(z^{-1}\right) \\
& -\frac{\epsilon}{1-\epsilon} F(z) S_{\overline{y y}}\left(z^{-1}\right)+\frac{\epsilon}{1-\epsilon} R_{\bar{y} \hat{y}}[0]+R_{\bar{y} y}[0] \\
= & H(z)\left(S_{\overline{y y}}\left(z^{-1}\right)+S_{\bar{y} w}\left(z^{-1}\right)\right)
\end{aligned}
$$

\subsection{Final results on PSDs}

\subsubsection{Computing $S_{\overline{y y}}(z)$}

Add eq. 34 and 35 together, we get

$$
\begin{aligned}
& (1-\epsilon)\left[S_{y y}^{+}(z)+S_{y y}^{+}\left(z^{-1}\right)\right]+\epsilon\left[S_{\hat{y} y}^{+}(z)+S_{y \hat{y}}^{+}\left(z^{-1}\right)\right]+\epsilon\left[S_{\hat{y} y}^{+}\left(z^{-1}\right)+S_{y \hat{y}}^{+}(z)\right]+\frac{\epsilon^{2}}{1-\epsilon}\left[S_{\hat{y} \hat{y}}^{+}(z)+S_{\hat{y} \hat{y}}^{+}\left(z^{-1}\right)\right] \\
& +\frac{1}{1-\epsilon}\left[S_{\overline{y y}}^{-}(z)+S_{\overline{y y}}^{-}\left(z^{-1}\right)\right]-\frac{\epsilon}{1-\epsilon}\left[F\left(z^{-1}\right) S_{\overline{y y}}(z)+F(z) S_{\overline{y y}}\left(z^{-1}\right)\right]+2 R_{\bar{y} y}[0]+\frac{2 \epsilon}{1-\epsilon} R_{\bar{y} \hat{y}}[0] \\
= & H\left(z^{-1}\right) S_{\overline{y y}}(z)+H(z) S_{\overline{y y}}\left(z^{-1}\right)+H\left(z^{-1}\right) S_{\bar{y} w}(z)+H(z) S_{\bar{y} w}\left(z^{-1}\right)
\end{aligned}
$$

We can simplify the above equation based on the following identities

$$
\begin{aligned}
S_{\overline{y y}}\left(z^{-1}\right) & =S_{\overline{y y}}(z) \\
S_{y y}^{+}(z)+S_{y y}^{+}\left(z^{-1}\right) & =S_{y y}(z)-R_{y y}[0] \\
S_{\hat{y} y}^{+}(z)+S_{y \hat{y}}^{+}\left(z^{-1}\right) & =S_{\hat{y} y}(z)-R_{\hat{y} y}[0] \\
S_{\hat{y} y}^{+}\left(z^{-1}\right)+S_{y \hat{y}}^{+}(z) & =S_{y \hat{y}}(z)-R_{y \hat{y}}[0] \\
S_{\hat{y} \hat{y}}^{+}(z)+S_{\hat{y} \hat{y}}^{+}\left(z^{-1}\right) & =S_{\hat{y} \hat{y}}(z)-R_{\hat{y} \hat{y}}[0] \\
S_{\bar{y} y}^{+}(z)+S_{\overline{y y}}^{+}\left(z^{-1}\right) & =S_{\overline{y y}}(z)-R_{\overline{y y}}[0]
\end{aligned}
$$

Then the simplified form is

$$
\begin{aligned}
& (1-\epsilon) S_{y y}(z)+\epsilon S_{\hat{y} y}(z)+\epsilon S_{\hat{y} y}\left(z^{-1}\right)+\frac{\epsilon^{2}}{1-\epsilon} S_{\hat{y} \hat{y}}(z)+\frac{1}{1-\epsilon} S_{\overline{y y}}(z) \\
& -\frac{\epsilon}{1-\epsilon}\left(F(z)+F\left(z^{-1}\right)\right) S_{\overline{y y}}(z)-\left(H(z)+H\left(z^{-1}\right)\right) S_{\overline{y y}}(z) \\
= & H(z) S_{\bar{y} w}\left(z^{-1}\right)+H\left(z^{-1}\right) S_{\bar{y} w}(z)+\Delta
\end{aligned}
$$


where

$$
\Delta=(1-\epsilon) R_{y y}[0]+\epsilon\left(R_{\hat{y} y}[0]+R_{y \hat{y}}[0]\right)+\frac{\epsilon^{2}}{1-\epsilon} R_{\hat{y} \hat{y}}[0]+\frac{1}{1-\epsilon} R_{\overline{y y}}[0]-\frac{2 \epsilon}{1-\epsilon} R_{\bar{y} \hat{y}}[0]-2 R_{\bar{y} y}[0]
$$

By $y=h *(\bar{y}+w)$ and $\hat{y}=f * \bar{y}$, we get

$$
\begin{aligned}
& S_{y y}(z)=H(z) H\left(z^{-1}\right)\left(S_{\overline{y y}}(z)+S_{\bar{y} w}(z)+S_{\bar{y} w}\left(z^{-1}\right)+S_{w w}(z)\right) \\
& S_{\hat{y} \hat{y}}(z)=F(z) F\left(z^{-1}\right) S_{\overline{y y}}(z) \\
& S_{\hat{y} y}(z)=F(z) S_{\bar{y} y}(z)=F(z) H\left(z^{-1}\right)\left(S_{\overline{y y}}(z)+S_{\bar{y} w}(z)\right)
\end{aligned}
$$

Substitute the above identities into eq. 36 , we get

$$
\begin{aligned}
& (1-\epsilon)\left[H(z) H\left(z^{-1}\right)+\frac{\epsilon}{1-\epsilon} F(z) H\left(z^{-1}\right)+\frac{\epsilon}{1-\epsilon} F\left(z^{-1}\right) H(z)+\frac{\epsilon^{2}}{(1-\epsilon)^{2}} F(z) F\left(z^{-1}\right)+\frac{1}{(1-\epsilon)^{2}}\right. \\
& \left.-\frac{\epsilon}{(1-\epsilon)^{2}}\left(F(z)+F\left(z^{-1}\right)\right)-\frac{1}{1-\epsilon}\left(H(z)+H\left(z^{-1}\right)\right)\right] S_{\overline{y y}}(z) \\
= & -(1-\epsilon) H(z) H\left(z^{-1}\right)\left(S_{\bar{y} w}(z)+S_{\bar{y} w}\left(z^{-1}\right)+S_{w w}(z)\right)-\epsilon F(z) H\left(z^{-1}\right) S_{\bar{y} w}(z) \\
& -\epsilon F\left(z^{-1}\right) H(z) S_{\bar{y} w}\left(z^{-1}\right)+H(z) S_{\bar{y} w}\left(z^{-1}\right)+H\left(z^{-1}\right) S_{\bar{y} w}(z)+\Delta
\end{aligned}
$$

The left side of the above equation can be simplified as follows

$$
\begin{aligned}
& (1-\epsilon)\left[H(z) H\left(z^{-1}\right)-H\left(z^{-1}\right) \frac{1-\epsilon F(z)}{1-\epsilon}-H(z) \frac{1-\epsilon F\left(z^{-1}\right)}{1-\epsilon}+\frac{1}{(1-\epsilon)^{2}}(1-\epsilon F(z))\left(1-\epsilon F\left(z^{-1}\right)\right)\right] \\
= & (1-\epsilon)\left(H(z)-\frac{1-\epsilon F(z)}{1-\epsilon}\right)\left(H\left(z^{-1}\right)-\frac{1-\epsilon F\left(z^{-1}\right)}{1-\epsilon}\right)
\end{aligned}
$$

Substitute eq. 23, 24 and 25 into the right side of eq. 41, it is simplified into

$$
(1-\epsilon) H(z) H\left(z^{-1}\right) S_{w w}(z)+\Delta
$$

So eq. 41 can be simplified into

$$
\begin{aligned}
& (1-\epsilon)\left(H(z)-\frac{1-\epsilon F(z)}{1-\epsilon}\right)\left(H\left(z^{-1}\right)-\frac{1-\epsilon F\left(z^{-1}\right)}{1-\epsilon}\right) S_{\overline{y y}}(z) \\
= & (1-\epsilon) H(z) H\left(z^{-1}\right) S_{w w}(z)+\Delta
\end{aligned}
$$

By the definition of $D(z)$, we get the final result on $S_{\overline{y y}}(z)$

$$
S_{\overline{y y}}(z)=\left|\frac{D(z) H(z)}{1-D(z) H(z)}\right|^{2} S_{w w}(z)+\frac{1}{1-\epsilon}\left|\frac{D(z)}{1-D(z) H(z)}\right|^{2} \Delta
$$

where $D(z)=\frac{1-\epsilon}{1-\epsilon F(z)}$.

\subsubsection{Computing $S_{y y}(z)$}

By eq. $38,23,42$, we can get

$$
S_{y y}(z)=\left|\frac{H(z)}{1-D(z) H(z)}\right|^{2} S_{w w}(z)+\frac{1}{1-\epsilon}\left|\frac{D(z) H(z)}{1-D(z) H(z)}\right|^{2} \Delta
$$




\subsubsection{Computing $\Delta$}

By $R_{\bar{y} y}[0]=(1-\epsilon) R_{y y}[0]+\epsilon R_{\hat{y} y}[0]$, we get

$$
\epsilon R_{\hat{y} y}[0]=R_{\bar{y} y}[0]-(1-\epsilon) R_{y y}[0]
$$

By $R_{\overline{y y}}[0]=(1-\epsilon) R_{y y}[0]+\epsilon R_{\hat{y} \hat{y}}[0]$, we get

$$
\epsilon R_{\hat{y} \hat{y}}[0]=R_{\overline{y y}}[0]-(1-\epsilon) R_{y y}[0]
$$

By $R_{\bar{y} \hat{y}}[0]=(1-\epsilon) R_{y \hat{y}}[0]+\epsilon R_{\hat{y} \hat{y}}[0]$ and $R_{y \hat{y}}[0]=R_{\hat{y} y}[0]$, we get

$$
R_{\bar{y} \hat{y}}[0]=\frac{1-\epsilon}{\epsilon}\left(R_{\bar{y} y}[0]-(1-\epsilon) R_{y y}[0]\right)+\left(R_{\overline{y y}}[0]-(1-\epsilon) R_{y y}[0]\right)
$$

Substitute eq. 44,45 and 46 , in addition to $R_{\bar{y} y}[0]=R_{y \bar{y}}[0]$, into eq. 37 , we get

$$
\Delta=\mathbf{E}\left[(y[n]-\bar{y}[n])^{2}\right]
$$

\subsubsection{Computing $S_{\tilde{y} \tilde{y}}(z)$}

By $\tilde{y}[n]=y[n]-\bar{y}[n]$, we get

$$
S_{\tilde{y} \tilde{y}}(z)=S_{y y}(z)+S_{\overline{y y}}(z)-S_{y \bar{y}}(z)-S_{\bar{y} y}(z)
$$

Substitute eq. $32,42,43$ and $S_{y \bar{y}}(z)=S_{\bar{y} y}\left(z^{-1}\right)$ into eq. 48 , we get

$$
S_{\tilde{y} \tilde{y}}(z)=\left|\frac{H(z)(D(z)-1)}{1-D(z) H(z)}\right|^{2} S_{w w}(z)+\frac{1}{1-\epsilon}\left|\frac{D(z)(1-H(z))}{1-D(z) H(z)}\right|^{2} \Delta
$$

Because $\Delta=R_{\tilde{y} \tilde{y}}[0]$,

$$
\Delta=\frac{1}{2 \pi} \int_{-\pi}^{\pi} S_{\tilde{y} \tilde{y}}\left(e^{j \omega}\right) d \omega
$$

\subsection{Existence, Uniqueness and Nonnegativity of the Solution to Equation 9}

By eq. 50 and 49 , we know $\Delta=R_{\tilde{y} \tilde{y}}[0]$ is a solution to eq. 9 . Because $R_{\tilde{y} \tilde{y}}[0] \geq 0$, eq. 9 has a nonnegative solution.

When $\epsilon>0$,

$$
\frac{1}{2 \pi} \int_{-\pi}^{\pi}\left|\frac{H\left(e^{j \omega}\right)\left(D\left(e^{j \omega}\right)-1\right)}{1-D\left(e^{j \omega}\right) H\left(e^{j \omega}\right)}\right|^{2} S_{w w}\left(e^{j \omega}\right) d \omega>0
$$

Then eq. 9 has a nonnegative solution if and only if

$$
\frac{1}{2 \pi} \int_{-\pi}^{\pi}\left|\frac{D\left(e^{j \omega}\right)\left(1-H\left(e^{j \omega}\right)\right)}{1-D\left(e^{j \omega}\right) H\left(e^{j \omega}\right)}\right|^{2} d \omega<1-\epsilon
$$

i.e. eq. 8 is valid. Furthermore, when eq. 8 is valid, eq. 9 has a unique solution.

Because $\Delta=R_{\tilde{y} \tilde{y}}[0]$ is a nonnegative solution to eq. 9 , the existence, uniqueness and nonnegativity of the solution to eq. 9 have all been established. $\diamond$ 


\section{$9 \quad$ Proof to Theorem 5.1}

In order to prove theorem 5.1, we need the following lemmas and corollaries.

Lemma 9.1 $\bar{A}_{[2]}=(1-\epsilon) A_{0}^{[2]}+\epsilon A_{1}^{[2]}$ is stable if and only if

$$
\exists P>0, \text { such that }(1-\epsilon) A_{0}^{T} P A_{0}+\epsilon A_{1}^{T} P A_{1}<P
$$

Proof: First we prove the necessary part. Obviously $\bar{A}_{[2]}$ is stable if and only if $\bar{A}_{[2]}^{T}=(1-\epsilon)\left(A_{0}^{T}\right)^{[2]}+\epsilon\left(A_{1}^{T}\right)^{[2]}$ is stable. Let $Q>0, P_{0}=Q$ and $P_{n+1}=(1-\epsilon) A_{0}^{T} P_{n} A_{0}+\epsilon A_{1}^{T} P_{n} A_{1}+Q(n \geq 0)$. It follows that

$$
P_{n+1} \geq P_{n}>0
$$

Apply the operator vec on the updation of $P_{n}$, we get

$$
\operatorname{vec}\left(P_{n+1}\right)=\bar{A}_{[2]}^{T} \operatorname{vec}\left(P_{n}\right)+\operatorname{vec}(Q)
$$

If $\bar{A}_{[2]}^{T}$ is stable, $\lim _{n \longrightarrow \infty} \operatorname{vec}\left(P_{n}\right)$ exists. Because $P_{n}=\operatorname{devec}\left(\operatorname{vec}\left(P_{n}\right)\right)$, vec and devec are all linear operators, we know that $\lim _{n \longrightarrow \infty} P_{n}$ exists. Let $P_{\infty}=\lim _{n \longrightarrow \infty} P_{n}$. Then $P_{\infty}$ is positive definite and satisfies the following equation

$$
(1-\epsilon) A_{0}^{T} P_{\infty} A_{0}+\epsilon A_{1}^{T} P_{\infty} A_{1}+Q=P_{\infty}
$$

Because $Q>0, P_{\infty}$ is a valid solution to eq. 52 .

Second we prove the sufficient part. If there exists $P>0$ such that $(1-\epsilon) A_{0}^{T} P A_{0}+\epsilon A_{1}^{T} P A_{1}<P$. We can construct a Lyapunov function $V(x)=x^{T} P x$ for system 4 . By eq. 52 , we know system 4 is asymptotically stable in mean square sense. Therefore $\bar{A}_{[2]}$ is stable. $\diamond$

Corollary 9.2 $\bar{A}_{[2]}=(1-\epsilon) A_{0}^{[2]}+\epsilon A_{1}^{[2]}$ is stable if and only if

$$
\exists P>0, \text { such that }(1-\epsilon) A_{0} P A_{0}^{T}+\epsilon A_{1} P A_{1}^{T}<P
$$

Proof: The same as the one of lemma 9.1 by replacing $A_{0}$ and $A_{1}$ respectively with $A_{0}^{T}$ and $A_{1}^{T} \cdot \diamond$

Lemma 9.3 Consider systems 4 and 11. For any symmetric matrix $P$,

$$
\left((1-\epsilon) A_{0} P A_{0}^{T}+\epsilon A_{1} P A_{1}^{T}\right)-\left(A_{e} P A_{e}^{T}\right)=\frac{1}{\epsilon(1-\epsilon)} B_{n} C_{\tilde{y}} P C_{\tilde{y}}^{T} B_{n}^{T}
$$

Proof: Based on the definitions of the matrices, we can easily get the results. $\diamond$

\subsection{Necessary Part of the Proof to Theorem 5.1}

The condition is that $\bar{A}_{[2]}=(1-\epsilon) A_{0}^{[2]}+\epsilon A_{1}^{[2]}$ is stable, so the original NCS 4 is asymptotically stable in mean square sense. Furthermore theorem 4.1 guarantees that eq. 9 has a unique nonnegative solution $\Delta_{N C S}=R_{\tilde{y} \tilde{y}}[0]$. 
By corollary 9.2 , there exist $P>0$ such that

$$
(1-\epsilon) A_{0} P A_{0}^{T}+\epsilon A_{1} P A_{1}^{T}<P
$$

Because $P>0, B_{n} C_{\tilde{y}} P C_{\tilde{y}}^{T} B_{n}^{T} \geq 0$. Lemma 9.3 leads to

$$
(1-\epsilon) A_{0} P A_{0}^{T}+\epsilon A_{1} P A_{1}^{T} \geq A_{e} P A_{e}^{T}
$$

Combine eq. 56 and 57, we get

$$
A_{e} P A_{e}^{T}<P
$$

So we get the stability of $A_{e}$, i.e. the stability of system 11. Because the input signals of system $11, w$ and $n$, are all WSS, all signals of system 11 , such as $y$ and $\tilde{y}$, are WSS. We can compute the related PSDs and expand eq. 10 by $R_{\tilde{y} \tilde{y}}[0]=\frac{1}{2 \pi} \int_{-\pi}^{\pi} S_{\tilde{y} \tilde{y}}\left(e^{j \omega}\right) d \omega$, which will be the same as eq. 9 . In the beginning we prove that $\Delta_{N C S}$ is the unique nonnegative solution to eq. 9. So $\Delta_{N C S}$ is also the unique nonnegative solution to eq. 10. Then eq. 10 is well-posed. Eq. 10 can be rewritten as

$$
\Delta=\left\|G_{\tilde{y} w}\right\|_{2}^{2}+\left\|G_{\tilde{y} n}\right\|_{2}^{2} \frac{1}{1-\epsilon} \Delta
$$

Because the above equation has a unique nonnegative solution with respect to $\Delta$ and $\left\|G_{\tilde{y} w}\right\|_{2}^{2} \geq 0$, we know constraint 12 is satisfied. For simplicity, we denote the common solution to eq. 9 and eq. 10 as $\Delta=\Delta_{N C S}$. Following the traditional control theories, we can compute $S_{y y}(z)$ and $S_{\tilde{y} \tilde{y}}(z)$ for system 11, which will be the same as eq. 5 and 6.

\subsection{Sufficient Part of the Proof to Theorem 5.1}

The condition is that $A_{e}$ is stable and $\left\|G_{\tilde{y} n}\right\|_{2}^{2}<1-\epsilon$. Then eq. 10 is well-posed. From eq. 13, we know

$$
\begin{aligned}
A_{e} W_{n} A_{e}^{T}+B_{n} B_{n}^{T} & =W_{n} \\
\left\|G_{\tilde{y} n}\right\|_{2}^{2} & =C_{\tilde{y}} W_{n} C_{\tilde{y}}^{T}+(1-\epsilon)^{2}
\end{aligned}
$$

Because $A_{e}$ is stable, there must exist a unique $P_{0}>0$ such that

$$
A_{e} P_{0} A_{e}^{T}+I=P_{0}
$$

where $I$ is the identity matrix with the appropriate dimension. Then $0 \leq C_{\tilde{y}} P_{0} C_{\tilde{y}}^{T}<\infty$.

$\left\|G_{\tilde{y} n}\right\|_{2}^{2}<1-\epsilon$ yields $C_{\tilde{y}} W_{n} C_{\tilde{y}}^{T}<\epsilon(1-\epsilon)$. By choosing a small positive number $\gamma(\gamma>0)$, we can guarantee $C_{\tilde{y}}\left(W_{n}+\gamma P_{0}\right) C_{\tilde{y}}^{T}<\epsilon(1-\epsilon)$. Let $P=W_{n}+\gamma P_{0}$. Then $P>0$ and $C_{\tilde{y}} P C_{\tilde{y}}^{T}<\epsilon(1-\epsilon)$. By lemma 9.3 , we get

$$
\begin{aligned}
& \left(A_{e} P A_{e}^{T}+B_{n} B_{n}^{T}\right)-\left((1-\epsilon) A_{0} P A_{0}^{T}+\epsilon A_{1} P A_{1}^{T}\right) \\
= & -\frac{1}{\epsilon(1-\epsilon)} B_{n} C_{\tilde{y}} P C_{\tilde{y}}^{T} B_{n}^{T}+B_{n} B_{n}^{T} \\
\geq & -B_{n} B_{n}^{T}+B_{n} B_{n}^{T} \\
= & 0
\end{aligned}
$$


So

$$
\begin{aligned}
(1-\epsilon) A_{0} P A_{0}^{T}+\epsilon A_{1} P A_{1}^{T} & \leq A_{e} P A_{e}^{T}+B_{n} B_{n}^{T} \\
& =A_{e} W_{n} A_{e}^{T}+B_{n} B_{n}^{T}+\gamma A_{e} P_{0} A_{e}^{T} \\
& =W_{n}+\gamma P_{0}-\gamma I \\
& <W_{n}+\gamma P_{0} \\
& =P
\end{aligned}
$$

Therefore there exists $P>0$ such that

$$
(1-\epsilon) A_{0} P A_{0}^{T}+\epsilon A_{1} P A_{1}^{T}<P
$$

By lemma 9.2 , we know $\bar{A}_{[2]}$ is stable. Then we can follow the similar procedure in the necessary part to show the two systems generate the same $S_{y y}(z), S_{\tilde{y} \tilde{y}}(z)$.

Then the proof is complete. $\diamond$

\section{References}

[1] N. Elia, S.K. Mitter, Stabilization of linear systems with limited information, IEEE Transactions on Automatic Control, Vol 46:1384-1400, 2001.

[2] H. Ishii and B.A. Francis, Quadratic stabiliation of sampled data systems with quantization, In Proc. 15th IFAC World Congress, Barcelona, 2002.

[3] G.N. Nair and R.J. Evans, Mean Square Stabilisability of Stochastic Linear Systems with Data Rate Constraints, Proceedings of the 41st IEEE Conference on Decision and Control, Las Vegas, Dec. 2002

[4] S. Tatikonda, A. Sahai, and S.K. Mitter, Control of LQG systems under communication constraints, In Proc., 37th IEEE Conference on Decision and Control, 1998.

[5] J. Nilsson. Real-time control systems with delays. PhD thesis, Lund Institute of Tecnology, 1998;

[6] W. Zhang. Stability analysis of networked control systems. PhD Thesis, Case Western Reserve University, 2001.

[7] C.N. Hadjicostis and R. Touri, "Feedback control utilizing packet dropping network links", IEEE Conference on Decision and Control, Las Vegas, Nevada, USA, December 2002.

[8] Q. Ling and M.D. Lemmon, Robust Performance of Soft Real-time Networked Control Systems with Data Dropouts, IEEE Conference on Decision and Control, Las Vegas, 2002.

[9] Q. Ling and M.D. Lemmon, Soft real-time scheduling of networked control systems with dropouts governed by a Markov chain, American Control Conference, Denver, Colorado, June 2003.

[10] P. Seiler, Coordinated Control of Unmanned Aerial Vehicles, Ph.D. Thesis, University of California, Berkeley, Mechanical Engineering, Fall 2001.

[11] E. Wong and B. Hajek. Stochastic Processes in Engineering Systems. Springer-Verlag, 1984.

[12] M. Mariton. Jump Linear Systems in Automatic Control. Marcel Dekker Inc., 1990. 
[13] H. Stark and J.W. Woods. Probability, Random Processes, and Estimation Theory for Engineers. Prentice Hall, Upper Saddle River, NJ, 1994. Second Edition.

[14] P.J. Antsaklis, A.N. Michel. Linear Systems. MacGraw-Hill, 1997.

[15] F. Kozin. "A survey of stability of stochastic systems", Automatica, vol. 5, pp.95-112, 1969. 